Wesen des sowjetischen Herrschaftssystems überzeugt werden. Der totalitäre Herrschaftscharakter des Stalinismus stand für sie fest. ${ }^{78}$

\title{
1.8 Die Gründung der CIA und ihre verdeckten Operationen
}

Während bereits der als ein weltweites Bollwerk zur Absicherung des amerikanischen Einflusses gedachte Marshallplan wesentlich auf den politischen Vorstellungen von Kennan beruhte, hatte der Direktor des Politischen Planungsstabes im Außenministerium außerdem einen erheblichen Anteil an der Gründung der CIA am 18. September 1947. Die Schaffung des ersten Geheimdienstes der Vereinigten Staaten zu »Friedenszeiten" stand hierbei ganz im Zeichen des antikommunistischen Grundkonsenses in der amerikanischen Führung und des heraufziehenden Kalten Krieges. Kennan wurde hierbei unterstützt von führenden Persönlichkeiten aus dem Außenministerium und dem diplomatischen Dienst wie Charles »Chip« Bohlen und Averell Harriman (also Exponenten der »Riga-Fraktion) sowie John McCloy, Joseph und Stewart Alsop, Walter Lippmann, Dean Acheson, George C. Marshall oder James Forrestal. Sie waren tonangebend in der US-amerikanischen Regierungspolitik und teilten nicht nur die Auffassungen über das Wesen des kommunistischen Regimes, sondern auch die Überzeugung, dass die Vereinigten Staaten eine Vorreiterrolle zu spielen hätten im Kampf der westlichen Demokratien gegen die Sowjetunion und ihre Satellitenstaaten. Sie hingen der für die außenpolitische Strategie im Kalten Krieg maßgeblichen traditionellen Vorstellung an, wonach antidemokratische Mächte auf Dauer eine Bedrohung der gewissermaßen natürlichen Interessen der Vereinigten Staaten darstellten. ${ }^{79}$ Ihr zentrales Thema war die Frage, wie die Übernahme Europas durch die Sowjetunion verhindert werden könne, und hierbei sollte neben dem Marshallplan der CIA eine entscheidende Funktion zufallen.

Mit der Unterschrift unter das Nationale Sicherheitsgesetz (National Security Act) am 26. Juli 1947 schuf Präsident Truman die gesetzliche Grundlage für die Gründung der CIA. Zudem wurde mit dem Gesetz der Nationale Sicherheitsrat (National Security Council, NSC) neugeschaffen, der als Vermittlungsstelle des Weißen Hauses für Entscheidungen des Präsidenten fungieren sollte. ${ }^{80}$ Dies entsprach den macht- und

78 Die amerikanische Öffentlichkeit wurde durch zwei weitere Buchveröffentlichungen über den tatsächlichen Charakter des sowjetischen Totalitarismus unterrichtet: vgl. Victor A. Kravchenko, I Chose Freedom, New York 1946 sowie David Dallin/Boris Nicolaevsky, Forced Labor in Soviet Russia, New Haven 1947. Die beiden Veröffentlichungen unterstrichen ein weiteres Mal, dass es sich beim ehemaligen Bündnispartner Sowjetunion um einen totalitären Polizeistaat handelte, und hoben nicht zuletzt die besondere Rolle des Ceheimdienstes NKWD hervor, durch die die sowjetische Bevölkerung eine extreme Unterdrückung und ganz spezifischen Grausamkeiten ausgesetzt sei. Zudem zeigten beide Veröffentlichungen, dass der NKWD das entscheidende Instrument war, um ein umfangreiches, weitverzweigtes Zwangsarbeitslagersystem mit Millionen von Gefangenen aufzubauen und am Leben zu erhalten und dass der sowjetischen Gesellschaft seinen Stempel aufdrückte. Beide Bücher besaßen innen- und außenpolitisch eine hohe Brisanz, weil es nicht nur der amerikanischen Öffentlichkeit ein weiteres Mal eindrucksvoll bestätigte, dass zwischen dem demokratischen System der Vereinigten Staaten und dem sowjetischen Totalitarismus ein fundamentaler Gegensatz bestand.

79 Stöver, Der Kalte Krieg 1947-1991, S. 21.

80 Weiner, CIA, S. 54. 
sicherheitspolitischen Erfordernissen der Vereinigten Staaten angesichts des Kalten Krieges und deckte sich mit dem in Gang gekommenen antikommunistischen Kurs. Angesichts der politischen Herausforderung, die die Sowjetunion darstellte, standen die Vereinigten Staaten indes vor einem besonderen Problem: Die Regierung hatte bekanntlich unmittelbar nach dem Ende des Zweiten Weltkrieges ihren Geheimdienst OSS größtenteils aufgelöst. ${ }^{81}$ Zudem war zum damaligen Zeitpunkt das Wissen über die Sowjetunion und Osteuropa nur rudimentär vorhanden. Insofern zählte es zu den dringendsten Aufgaben der CIA, in möglichst kurzer Zeit möglichst erstklassige Geheiminformationen über die Sowjetunion zu gewinnen und im Grunde genommen aus dem Nichts heraus ein koordiniertes System der Informationsgewinnung zu errichten. ${ }^{82}$ Im Gegensatz hierzu verfügte der sowjetische Staat über einen seit fast 30 Jahren unter verschiedenen Namen operierenden Geheimdienstapparat, der sowohl im In- als auch im Ausland unter Beweis gestellt hatte, dass er sein »Handwerk« verstand.

Für das demokratische Verfassungssystem der Vereinigten Staaten und die amerikanische Öffentlichkeit war die Schaffung der CIA indes von Anfang an mit Problemen behaftet. Dies betraf insbesondere Fragen der demokratischen Legitimation sowie der Kontrolle des Geheimdienstes und resultierte insbesondere aus ihren unzureichend definierten Machtbefugnissen. Knapp zwei Jahre lang gab es weder eine vom Kongress abgesegnete verfassungsmäßige Satzung, d.h., sie operierte außerhalb der US-amerikanischen Gesetze, noch rechtlich abgesicherte Geldmittel, sodass in dieser Zeit die CIA-Zentrale nur aufgrund eines Unterstützungsfonds überleben konnte, den einige wenige Kongressmitglieder ins Leben gerufen hatten. Und die von der CIA praktizierte Geheimhaltung, die immer wieder in Konflikt mit der Offenheit der amerikanischen Demokratie geriet, machte sogar führenden Regierungspolitikern frühzeitig Sorge. So schrieb der spätere amerikanische Außenminister Dean Acheson: "Diese Organisation ließ mich das Schlimmste befürchten, und ich wies den Präsidenten darauf hin, dass - so wie sie angelegt war - weder er noch der Nationale Sicherheitsrat noch sonst irgendwer jemals in der Lage sein würde, zu erfahren, was sie tut, oder sie zu kontrollieren. $\ll^{83}$

Laut Gesetz war die CIA als ein Geheimdienst im traditionellen Sinne geplant, deren vornehmliche Aufgabe im Sammeln, Auswerten und der Weitergabe von sicherheitsrelevanten Informationen bestand. Zudem sollte sie den Nationalen Sicherheitsrat beraten und schließlich »solch andere Aufgaben und Pflichten wahr[...]nehmen, die nachrichtendienstliche Aktivitäten betreffen, welche die nationale Sicherheit berühren und von Zeit zu Zeit vom Nationalen Sicherheitsrat angeordnet werden ${ }^{84}$. An

81 Zur Gründung der CIA vor dem Hintergrund der Auflösung des OSS-Apparates vgl. Thomas F. Troy, Wild Bill and Intrepid: Donovan, Stephenson, and the Origin of the CIA, New Haven: Yale University Press 1996.

82 Die wenigen Erkenntnisse, über die Washington damals mit Blick auf das sowjetische Herrschaftsregime und die kommunistische Ideologie verfügte, stammten u. a. von dem Russlandexperten Kennan.

83 Zit. n. Weiner, ClA, S. 54.

84 Zit. n. Gebhard Schweigler, Institutionen, Entscheidungsprozesse und Instrumente der Außenpolitik, in: Willi Paul Adams u. a. (Hg.), Länderbericht USA. Bd. 2: Außenpolitik/Gesellschaft/Kultur-Religion-Erziehung, 2., aktual. und erg. Aufl., Bonn 1992, S. 3-31, hier S. 15. 
eigene geheime Aktivitäten der CIA in ausländischen Staaten war ursprünglich im Gesetz weniger gedacht, wobei sich allerdings sehr schnell herausstellte, dass diese Gesetzeslücke verdeckte Geheimoperationen ermöglichte und insofern eine scharfe Trennung zwischen dem nachrichtendienstlichen und geheimdienstlichen Aktionsfeld nicht aufrechterhalten wurde. Andererseits zeigte sich bei der Durchführung der verdeckten Operationen, die nur mit direkter oder stillschweigender Genehmigung des Nationalen Sicherheitsrates möglich waren, dass die CIA nach dem Motto agierte, wonach zur Abwehr der kommunistischen Gefahr der Zweck die Mittel heiligt und rechtsstaatliche Grundsätze außer Kraft gesetzt werden konnten. Es herrschte die Überzeugung vor, dass im Umgang mit einem gefährlichen Gegner, der sich selbst an keinerlei (demokratische) Spielregeln hielt, auch die eigene Seite solche Regeln missachten dürfte.

Vor dem Hintergrund von Kennans politischen Vorstellungen stellte der Nationale Sicherheitsrat der CIA innerhalb von wenigen Monaten zwei Direktiven für geheime Operationen aus, die fortan richtungsweisend waren für den amerikanischen Geheimdienst auf dem Feld der politischen Kriegsführung. In der ersten Direktive (NSC-4) vom 19. Dezember 1947 wurde der CIA-Direktor in einem streng geheimen Anhang (NSC-4A) angewiesen, »verdeckte psychologische Aktivitäten« zur Abwehr sowjetischer und sowjetisch gesteuerter Aktivitäten durchzuführen. Da indes unklar blieb, welche Verfahren zur Koordinierung bzw. Genehmigung solcher Aktivitäten befolgt werden sollten, wurde sie am 18. Juni 1948 durch eine neue, eindeutiger formulierte Direktive ersetzt (NSC-10/2), die die CIA ermächtigte, ein breites Spektrum von Geheimoperationen durchzuführen: »Dazu zählten Propaganda, Wirtschaftskrieg, Präventivmaßnahmen einschließlich Sabotage, Gegensabotage, Zerstörung und Evakuierung, ferner subversives Vorgehen gegen feindliche Staaten einschließlich der Unterstützung von Widerstandsbewegungen, Guerilla und Flüchtlingsbewegungen. $\aleph^{85}$ Allerdings wurde die CIA dazu verpflichtet, sämtliche Geheimoperationen, von denen es bald Hunderte gab - 81 bereits in der zweiten Amtsperiode Trumans ${ }^{86}$ - so durchzuführen, dass sich die amerikanische Regierung zu jedem Zeitpunkt glaubhaft davon distanzieren könne; dies implizierte immer wieder verfassungsrechtliche und politische Probleme für den Präsidenten, der für die Tätigkeiten des Geheimdienstes und mithin auch für illegale Aktivitäten letztlich die Verantwortung trug.

Außerdem sah die Direktive NSC-10/2 die Einrichtung einer speziellen Abteilung für verdeckte Aktionen (covert actions) vor, die zwar in die CIA integriert wurde, jedoch politisch und personell dem Planungsstab des Außenministeriums unterstellt war. Diese »Eingreiftruppe« (Tim Weiner) fungierte schließlich unter dem Namen Office of Policy Coordination (Büro für politische Koordination; OPC) - ein Deckname, mit dem die Arbeit dieser Gruppe kaschiert werden sollte. ${ }^{87}$ Verdeckte Aktionen wurden wie folgt definiert: jede "geheime Handlung mit dem Ziel der Beeinflussung von ausländischen Regierungen, Veranstaltungen, Organisationen oder Personen zur Unterstützung der amerikanischen Außenpolitik. Diese Aktivitäten sind so auszuführen, dass die Beteiligung der amerikanischen Regierung nicht sichtbar wird. ${ }^{88}$ Zum Leiter

85 Saunders, Wer die Zeche zahlt ..., S. 48 f.

86 So Weiner, CIA, S. $54 \mathrm{f}$.

87 Ebd., S. 60.

88 Zit. n. Saunders, Wer die Zeche zahlt ..., S. 49. 
dieser neuen Abteilung, die am 1. September 1948 ihre Arbeit aufnahm, wurde, wie bereits gesagt, der ehemalige OSS-Mitarbeiter und stellvertretende Leiter der Außenamtsabteilung für die besetzenden Gebiete Frank G. Wisner ernannt, der nicht zufällig aus der »Kennan-Gruppe«stammte.

Der amerikanische Kongress wiederum setzte dieser gesamten Entwicklung, die Schritt für Schritt mit einem starken personellen und finanziellen Ausbau der CIA korrespondierte, keinen Widerstand entgegen. Im Gegenteil: um dem OPC einen größeren Handlungsspielraum einzuräumen, erließ er 1949 den Central Intelligence Agency Act, der die CIA ermächtigte, »im Gegensatz zu verfassungsrechtlichen Bestimmungen, die die Offenlegung und ausdrückliche Bewilligung aller Regierungsausgaben verlangen, ihre Mittel geheim und ohne Verpflichtung zur Rechenschaftslegung« zu verwenden. Damit war ein wesentliches Instrument »kongressioneller - und gleichzeitig auch öffentlicher - Kontrolle« aus »der Hand gegeben worden «. ${ }^{89}$

Zur eigentlichen Geburtsstunde der verdeckten Operationen im Kalten Krieg wurden die italienischen Parlamentswahlen im April 1948. Am 14. Dezember 1947 erteilte der Nationale Sicherheitsrat die Anweisung, mit schätzungsweise zehn Millionen Dollar die demokratischen Parteien, zumal die Christdemokraten, gegen die Kommunisten in Italien zu unterstützen. ${ }^{90} \mathrm{Da}$ die CIA damals noch immer über keinen eigenen Etat verfügte, wurden Gelder aus dem für den Wiederaufbau Europas vorgesehenen Währungsstabilitätsfonds abgezweigt und an von der CIA gegründete Tarnorganisationen weitergeleitet. ${ }^{91}$ Nicht nur aus diesem Grund fehlte der verdeckten Operation, die nicht vom Kongress abgesegnet wurde, die demokratische Legitimität. Sie begann schon Wochen vor der offiziellen Genehmigung durch den Nationalen Sicherheitsrat, und insofern war diese CIA-Aktion, die zum Beispiel auch die Ermordung von führenden kommunistischen Politikern und Gewerkschaftsfunktionären beinhaltete, schlichtweg illegal..$^{92}$ Gleichwohl konnten die Verantwortlichen mit dem Ergebnis zufrieden sein. Obwohl die sowjetische Seite ebenfalls im italienischen Wahlkampf ak-

89 Schweigler, Institutionen, Entscheidungsprozesse und Instrumente der Außenpolitik, S. 16.

90 Für die in der amerikanischen Führung und speziell dem Nationalen Sicherheitsrat vorherrschende Auffassung stand die von Kennan stellvertretend. Es sei besser, so Kennan, einen »heißen Krieg zu führen, als zuzulassen, dass die Kommunisten legal die Macht übernehmen - aber zweitbeste Wahl seien verdeckte Aktionen nach dem Muster der kommunistischen Wühlarbeit« (Weiner, CIA, S. 56).

91 Weiner beschreibt diesen Vorgang - indes ohne Quellenangabe - in seiner CIA-Studie wie folgt: Demnach konnte James Forrestal den damaligen Finanzminister und einen der engsten Mitstreiter von Präsident Truman, John W. Snyder, dazu überreden, den Währungsstabilitätsfonds anzuzapfen, in dem sich zum damaligen Zeitpunkt 200 Millionen Dollar befanden, die für den Wiederaufbau Europas bestimmt waren. »Von dort«, so Weiner, »flossen nun Millionen Dollar auf die Bankkonten reicher amerikanischer Staatsbürger, viele von ihnen Italo-Amerikaner, die die überwiesenen Summen ihrerseits an neue, von der CIA gegründete Tarnorganisationen weiterleiteten. Diese Spender wurden aufgefordert, auf ihrer Einkommenssteuererklärung neben der sSpende zu wohltätigen Zwecken< einen Code einzutragen. Millionen wurden an italienische Politiker und an die `Katholische Aktion<, einen politischen Arm des Vatikans, verteilt. Koffer voller Bargeld wechselten im Viersternehotel Hassler den Besitzer.« Der damals involvierte CIA-Mitarbeiter F. Mark Wyatt kommentiert dies später mit folgenden Worten: »Wir hätten es gerne etwas raffinierter eingefädelt [...] mit Schmiergeld politische Wahlen beeinflussen, das hat nicht gerade Stil« (zit. n. ebd., S. 57).

92 Dies war den Verantwortlichen auch bewusst. So sagte einer der CIA-Mitarbeiter im Nachhinein: »In der CIA-Zentrale waren wir einfach entsetzt, zu Tode erschrocken. Wir überschritten die Grenzen unseres Statuts« (zit. n. ebd., S. 56). 
tiv war, gewannen die Christdemokraten mit ausreichendem Vorsprung und bildeten eine Regierung ohne die Kommunisten.

Nachdem im April 1948 die politische Entwicklung in Italien zugunsten der Vereinigten Staaten entschieden worden war, war sich die amerikanische Regierung indessen darüber im Klaren, dass der Hauptschauplatz im Kalten Krieg in Deutschland und speziell in Berlin lag. Bereits im Sommer 1945 hatten die Vereinigten Staaten im geteilten Berlin ein Geheimdienstbüro eingerichtet, um Informationen über die Sowjetische Besatzungszone zu sammeln und wo sich nun der amerikanische und sowjetische Geheimdienst mit allen Mitteln der Spionage und Gegenspionage bekämpften. ${ }^{93}$ Das OPC, das sich unter der Führung von Wisner zur am schnellsten wachsenden Abteilung der CIA entwickelte, errichtete auf dem Gelände des Militärflughafens Berlin-Tempelhof ein Büro. Denn der Beginn der sowjetischen Blockade Westberlins am 24. Juni 1948 hatte die Vereinigten Staaten massiv unter Druck gesetzt und nach Ansicht der CIA-Führung die Gefahr einer offenen militärischen Konfrontation konkret werden lassen. Dieses Ereignis löste einen beispiellosen Ausbau der verdeckten Operationen der CIA aus, die hinsichtlich der gesamten »Ostarbeit« des amerikanischen Geheimdienstes darauf ausgelegt waren, das gegnerische System zu schwächen und das eigene zu stärken. Waren die Vereinigten Staaten und die Sowjetunion im Zweiten Weltkrieg noch verbündete im Kampf gegen den Nationalsozialismus, machte der CIA nun in der weltweiten Auseinandersetzung mit dem Kommunismus mit zahlreichen rechtsgerichteten Diktaturen sowie Personen und Gruppen aus dem nationalsozialistischen bzw. faschistischen Lager gemeinsame Sache. Mit Blick auf die Bundesrepublik Deutschland bedeutete das zum Beispiel, dass der CIA-Apparat im Juli 1949 die Organisation Gehlen von der US-Armee übernahm - die hatte bereits im Sommer 1945 auf General Reinhard Gehlen, den Leiter der Abteilung fremde Heere Ost in Hitlers Generalstab, zurückgriffen, um Informationen über die Sowjetunion zu gewinnen -, zu dessen Personal auch Dutzende prominenter Kriegsverbrecher zählten, und machte sich daran, sie in einen westlichen Nachrichtendienst umzuwandeln. Unter der Leitung von Gehlen wurde sie 1956 zum Bundesnachrichtendienst (BND) der Bundesrepublik Deutschland. Zudem unterstützte die CIA mit Blick auf den westlichen Teil Deutschlands die knapp 20000 Mitglieder starke rechtsradikale Gruppe Bund Deutscher Jugend (BDJ). ${ }^{94}$

Einen wichtigen Teil der verdeckten Operationen der CIA machten die Umsturzversuche und Unterstützung von antikommunistischen »Rebellen« hinter dem Eisernen Vorhang aus. Die ab 1949 vom amerikanischen Geheimdienst initiierten direkten Putschversuche liefen hierbei immer nach dem gleichen Muster ab. In der Regel wurden Emigranten aus den kommunistischen Staaten ausgebildet und für die Auslösung eines Umsturzes eingesetzt. Bis weit in die 1950er-Jahre gab es Umsturzversuche (u. a. in Jugoslawien, in der Ukraine, in den Baltischen Staaten und im Kaukasus) und Bemühungen, in der Sowjetunion antikommunistische Zellen ins Leben zu rufen, die

93 Vgl. hierzu grundsätzlich Ceorge Bailey, Sergej A. Kondraschow, David E. Murphy, Die unsichtbare Front. Der Krieg der Geheimdienste im geteilten Berlin, Berlin 1997.

94 Die»Partisaneneinheit «wurde u. a. mit militärischem Material ausgestattet, das für einen möglichen Ernstfall im ganzen Land vergraben wurde. Zudem erstellte der Bund Deutscher Jugend eine umfangreiche Liste mit Namen von westdeutschen Politikern, die getötet werden sollten, wenn die Zeit reif wäre. Siehe Weiner, CIA, S. 106. 
schließlich wegen kontinuierlicher Erfolglosigkeit eingestellt wurden. In die gleiche Richtung zielte die sogenannte Psychologische Kriegsführung in den sowjetisch kontrollierten Staaten in Ostmitteleuropa, mit der das Ziel verfolgt wurde, die kommunistischen Systeme von innen $\mathrm{zu}$ untergraben, $\mathrm{zu}$ destabilisieren und damit einen Regierungswechsel vorzubereiten. Hierzu dienten beispielsweise halbprivate amerikanische Rundfunksender wie Radio Free Europe oder Radio Liberation, die in den kommunistischen Machtbereich ausstrahlten und zu den vielgehörten Stationen gehörten. Zudem wurden in vielen Staaten hinter dem Eisernen Vorhang Flugblattaktionen durchgeführt, die, sobald sich eine latente Unzufriedenheit in der Bevölkerung zeigte, verstärkt wurden. An diesen Aktionen beteiligten sich auch teils private und höchst militante Organisationen. Einige wie die von der CIA 1949 indirekt unterstützte und ab 1950 direkt finanzierte Berliner Kampfgruppe gegen Unmenschlichkeit führten auch selbst Anschläge in der DDR durch. ${ }^{95}$

Insofern bildete, wie gesagt, der weltweit ausgerichtete »Kongreß für kulturelle Freiheit« im Rahmen der verdeckten CIA-Operationen einen wichtigen Bestandteil eines Netzwerkes von Gruppen und Organisationen im Kalten Krieg, ein »Geflecht höchst unterschiedlicher Agenturen mit ebenso mannigfaltigen Aufgabenbereichen innerhalb des Gesamtzusammenhanges der Auseinandersetzung zwischen den liberaldemokratischen Staaten des Westens und der kommunistischen Welt $\aleph^{96}$. In diesem Kontext verfolgten der »Kongreß « und die mit ihm organisatorisch und personell eng verbundene Zeitschrift Der Monat, wie zu zeigen sein wird, mit Blick auf den deutschen Sprach- und Kulturraum ganz spezifische Ziele.

\section{Die Deutschlandfrage im Schatten des Kalten Krieges}

\subsection{Grundzüge der Deutschlandpolitik bei den Alliierten während des Zweiten Weltkrieges}

Der deutsche Überfall auf die Sowjetunion am 22. Juni 1941 und die Kriegserklärung Hitlers an die Vereinigten Staaten am 11. Dezember desselben Jahres führte in Anbetracht der existenziellen Bedrohung nicht nur der europäischen Staaten zu der Anti-Hitler-Koalition, bestehend aus den drei Weltmächten Großbritannien, der Sowjetunion und den USA. Spätestens im Sommer 1943 zeichnete sich ab, dass der nationalsozialistische Versuch, die Utopie einer nach rassistischen Kategorien hierarchisierten Neuordnung Europas unter der Führung Deutschlands zu realisieren, zum Scheitern verurteilt war. Nicht nur die nach den militärischen Erfolgen im Zuge der deutschen Besatzungspolitik begangenen, ideologisch motivierten Verbrechen an den jüdischen und nicht jüdischen Zivilisten in der Sowjetunion und Polen ließ die Alliierten zu der Überzeugung kommen, dass der Zweite Weltkrieg mit der bedingungslosen Kapitulation Deutschlands beendet werden muss. Nur durch die gemeinsame Anstrengung der alliierten Streitkräfte war es am Ende gelungen, den nationalsozialistischen Aggressor nicht nur aufzuhalten, sondern schließlich zu besiegen. Seit Dezember 1941 stand die Frage, wie man mit dem Problem Deutschland nach Kriegsende

95 Siehe Stöver, Der Kalte Krieg 1947-1991, S. 28 ff.

96 So Hochgeschwender, Freiheit in der Offensive?, S. 17. 